\title{
Obtaining and investigation of the conditions of heat transfer through inhomogeneous inclusion with heat sources
}

\author{
Gera B. V. ${ }^{1}$, Dmytruk V. A. ${ }^{2,3}$ \\ ${ }^{1}$ Dnipropetrovsk National University of Railway Transport named after academician V. Lazaryan \\ 12a I. Blazhkevych str., 79052, Lviv, Ukraine \\ ${ }^{2}$ Centre of Mathematical Modelling of Ukrainian National Academy of Sciences \\ 15 D. Dudayev str., 79005, Lviv, Ukraine \\ ${ }^{3}$ Lviv Polytechnic National University \\ 12 S. Bandera str., 79013, Lviv, Ukraine
}

(Received 27 March 2015)

\begin{abstract}
The conditions of nonideal heat transfer are obtained for a heat conduction problem of contacting layers through a thin heterogeneous sublayer (inclusion) with acting heat sources (heat sinks). These conditions include both thermal resistances of components of the inclusion and specific powers of heat sources in them. On this basis, the heat conduction problem with nonideal conditions of heat transfer without consideration of inner inclusion is written down. Their use ensures observable features of temperature and heat flux, i.e. the function of temperature and the function of heat flux have a drop when passing through the contact interface. The magnitude of thermal resistance is suggested to be determined by means of more usual for measuring values, e.g. a thermal conductivity coefficient and a thickness of the inclusion. Surface specific heat sources can be set if the values of heat dissipation on the contact boundary are known. From the obtained and generalized heat transfer conditions, the known conditions of nonideal thermal contact can be obtained as particular cases.
\end{abstract}

Keywords: heat conduction problem, composite layer, thin inclusion, heat transfer, nonideal contact conditions

2000 MSC: 80A20; 93A30; 35K05

UDC: $536.2: 539.3$

\section{Introduction}

In mathematical modeling of the conditions of thermal interaction between the contacting bodies, in many cases the nonideal contact conditions are used [1-3]. Modeling these conditions involves representing the peculiarities of transmission of heat through rough surfaces, connections of bodies through intermediate thin inclusions, which have a much lower thermal conductivity resulting in local temperature gradients. If there is a heat dissipation in the contact zone, it is also noticed a distinguishable change in the heat flux. Consideration of such effects is possible due to the introduction of additional parameters into the conditions of heat transfer, which must ensure the jump of temperature and heat flux in the zone of interface transition.

To determine the value of parameters included in the nonideal conditions of thermal contact is possible by the solutions of the corresponding inverse problems [4-7], as well as by examining bodies of layered structure, introducing the contact zone as a thin intermediate inclusion between layers [8]. If the inclusion creates heat insulating effect and the values of its thermal characteristics, in particular the conductivity, significantly differ from the characteristics of the contacting layers, then a significant temperature drop is observed in it, which in the mathematical model can be approximately introduced by a jump function. The conditions of the nonideal heat transfer are obtained by directing the thickness 
of the inclusion to zero keeping its impact on heat transfer, which is achieved due to the presence of the contact thermal resistance in the heat exchange conditions $[9,10]$.

The conditions of heat transfer can have a more complicated form if on the contact interface another physical, mechanical, and chemical processes occur, what leads, in particular, to the appearance of heat sources. Power of heat sources should be estimated and taken into account in the conditions of heat exchange. This can lead also to the need to use mathematical modeling of interconnected thermophysical processes. Thus we obtain the more general conditions of thermal contact, such as Barber-Protasov's ones [11-13]. The solving of a heat conduction problem with such conditions allows us to obtain the jump of the heat flux in the zone of the interface transition, which is observed experimentally and used in several papers $[4,14-16]$.

In this article, for heat conduction problems of contacting layers through a thin inclusion, in which heat sources (sinks) are acting, the nonideal conditions of heat transfer are obtained. Besides, there is considered a case when these conditions contain the thermal resistance of the inclusion as well as specific power of heat sources, allowing us to write down the heat conduction problem without consideration of the intermediate inclusion but with the nonideal conditions of heat transfer. Their use ensures obtaining observable features of temperature and a heat flux, namely, the function of temperature and a heat flux are subjected a jump change when passing through the interface. The value of heat resistance is suggested to be estimated by means of more usual for measuring quantities - coefficients of heat conductivity and the thickness of the inclusion. Surface specific heat sources can be defined if the values of heat dissipation on the interface are known. Also the solution of the heat conduction problem under the non-ideal conditions of heat exchange is obtained.

\section{Heat transfer through thin inclusion under constant action of heat sources}

To obtain the conditions of the non-ideal thermal contact in a heterogeneous body, let us consider a conductive layer made up of two layers (1) and (2), which are contacting through a thin inclusion (3) (Fig. 1). With the help of the inclusion, thermophysical characteristics of which differ from the characteristics of the main layers we simulate the effect of the interface on the characteristics of heat transfer between the parts of the body. Consider the piecewise uniform conductive layer of the thickness $L$ composed of two layers, occupying the domain $\left(0 ; z_{1}\right)$ and $\left(z_{2} ; L\right)$ contacting through a thin inclusion of the thickness $h=z_{2}-z_{1}$.

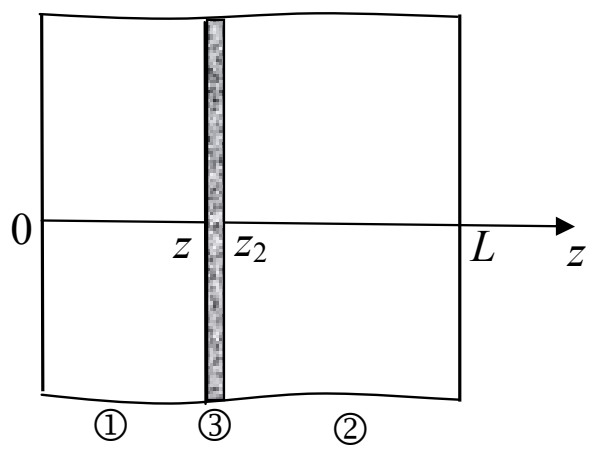

Fig. 1. Heat-conducting layer with a thin intermediate inclusion of the thickness $h$.
Describe the process of heat conduction in the layer, if in the layer $(3$ starting from an instant of time constant heat sources of $Q_{*}$ power have been acting. On the external surfaces $z=$ $=0$ and $z=L$ we assume the temperature to be known, and on the internal ones $z=z_{1}$ and $z=$ $=z_{2}$ we impose the conditions of ideal thermal contact, i.e. equality of heat fluxes and temperature from both sides. Generalized thermophysical characteristics of the inclusion that depend on the way and technology of the layers connection and inner surfaces quality define a heat exchange between the main layers (1) and (2). Coefficients of conductivity and specific heat of the layers (1), (2) are denoted as $\lambda_{1}, c_{1}$ and $\lambda_{2}$, $c_{2}$, and the inclusions (3) as $\lambda_{3}, c_{3}$. The function of the temperature $t(z, \tau)$ of the coordinate and the time for each of the layers we also denote by the corresponding index.

Mathematical Modeling and Computing, Vol. 2, No. 1, pp. 33-47 (2015) 
The heat equation in domains $\Omega_{1}=\left(0 ; z_{1}\right)$ and $\Omega_{2}=\left(z_{2} ; L\right)$ is written as follows

$$
c_{i} \frac{\partial t_{i}(z, \tau)}{\partial \tau}-\lambda_{i} \frac{\partial^{2} t_{i}(z, \tau)}{\partial z^{2}}=0, \quad z \in \Omega_{i} \quad(i=1 ; 2), \quad \tau>0
$$

and in the inclusion

$$
c_{3} \frac{\partial t_{3}(z, \tau)}{\partial \tau}-\lambda_{3} \frac{\partial^{2} t_{3}(z, \tau)}{\partial z^{2}}=Q_{*}, \quad z \in\left(z_{1} ; z_{2}\right), \quad \tau>0 .
$$

Here $t_{i}(z, \tau)$ is the temperature function $(i=1 ; 2 ; 3), z$ is the coordinate, $\tau$ is the time, $Q_{*}$ is the specific power of heat sources.

At the initial time, the temperature distribution is given as

$$
t_{i}(z, 0)=\varphi_{i}(z) \quad(i=1 ; 2 ; 3) .
$$

On the surfaces $z=0$ and $z=L$, the values of temperature are known

$$
t_{1}(0, \tau)=f_{0}, \quad t_{2}(L, \tau)=f_{L}
$$

On the internal surfaces $z=z_{1}$ and $z=z_{2}$ we write down the conditions of ideal thermal contact

$$
\begin{aligned}
& \left.\lambda_{1} \frac{\partial t_{1}(z, \tau)}{\partial z}\right|_{z=z_{1}-0}=\left.\lambda_{3} \frac{\partial t_{3}(z, \tau)}{\partial z}\right|_{z=z_{1}+0},\left.\quad t_{1}(z, \tau)\right|_{z=z_{1}-0}=\left.t_{3}(z, \tau)\right|_{z=z_{1}+0} ; \\
& \left.\lambda_{3} \frac{\partial t_{3}(z, \tau)}{\partial z}\right|_{z=z_{2}-0}=\left.\lambda_{2} \frac{\partial t_{2}(z, \tau)}{\partial z}\right|_{z=z_{2}+0},\left.\quad t_{3}(z, \tau)\right|_{z=z_{2}-0}=\left.t_{2}(z, \tau)\right|_{z=z_{2}+0} ;
\end{aligned}
$$

For thin-walled structural elements there are often used the approximations of linear temperature distribution with respect to the thickness [8]. Allowing for the small thickness of the inclusion, we also use the approximate representation of its temperature function $t_{3}(z, \tau)$ and accounting for the heat sources act we write it in the following form

$$
t_{3}\left(z^{\prime}, \tau\right)=T_{1}(\tau)+\frac{z^{\prime}}{h} T_{2}(\tau)-\frac{Q_{*}}{\lambda_{3}} \frac{z^{\prime 2}}{2},
$$

where $T_{1}(\tau)$ and $T_{2}(\tau)$ are the reduced temperature and temperature instant of the inclusion, $z^{\prime}=z-\left(z_{1}+z_{2}\right) / 2$ is the changed coordinate with respect to the thickness, so that the origin is moved to the middle of the inclusion.

To determine $T_{1}(\tau)$ and $T_{2}^{\prime}(\tau)$, let us use the known formula [8]

$$
T_{1}(\tau)=\frac{1}{h} \int_{-h / 2}^{h / 2} t(z, \tau) d z, \quad T_{2}=\frac{12}{h^{2}} \int_{-h / 2}^{h / 2} t(z, \tau) z d z .
$$

The last term of (7) ensures the fulfillment of the heat equation with heat sources. Substituting (7) in the conditions (5), (6) of thermal contact on the internal surfaces, we have

for $z=z_{1}$

$$
t_{2}\left(z_{2}, \tau\right)=T_{1}(\tau)-\frac{1}{2} T_{2}(\tau)-\frac{Q_{*}}{\lambda_{3}} \frac{h}{8} ;\left.\quad \lambda_{1} \frac{\partial t_{1}(z, \tau)}{\partial z}\right|_{z=z_{1}}=\frac{\lambda_{3}}{h} T_{2}(\tau)+\frac{Q_{*} h}{2} ;
$$


for $z=z_{2}$ :

$$
t_{2}\left(z_{2}, \tau\right)=T_{1}(\tau)+\frac{1}{2} T_{2}(\tau)-\frac{Q_{*}}{\lambda_{3}} \frac{h}{8} ;\left.\quad \lambda_{2} \frac{\partial t_{2}(z, \tau)}{\partial z}\right|_{z=z_{2}}=\frac{\lambda_{3}}{h} T_{2}(\tau)+\frac{Q_{*} h}{2}
$$

From the four conditions (8), (9) we exclude $T_{1}(\tau)$ and $T_{2}(\tau)$. We obtain two conditions

$$
\begin{aligned}
& \left.\lambda_{1} \frac{\partial t_{1}(z, \tau)}{\partial z}\right|_{z=z_{1}}=\frac{\lambda_{3}}{h}\left(t_{2}\left(z_{2}, \tau\right)-t_{1}\left(z_{1}, \tau\right)\right)+\frac{Q_{*} h}{2}, \\
& \left.\lambda_{2} \frac{\partial t_{2}(z, \tau)}{\partial z}\right|_{z=z_{2}}=\frac{\lambda_{3}}{h}\left(t_{2}\left(z_{2}, \tau\right)-t_{1}\left(z_{1}, \tau\right)\right)+\frac{Q_{*} h}{2} .
\end{aligned}
$$

Note that with this $T_{2}(\tau)=t_{2}\left(z_{2}, \tau\right)-t_{1}\left(z_{1}, \tau\right)$, i.e. $T_{2}(\tau)$ has the value of the temperature drop on the surfaces of the inclusion.

We introduce the notations in the conditions of (10), (11)

$w=Q_{*} h$ is the specific power of the surface heat sources,

$R=\frac{h}{\lambda_{3}}$ is the contact thermal resistance.

Instead one of the conditions (10), (11), let us take their sum and write down contact conditions, which take into account the impact of the inclusion on the temperature, but have no visibly $t_{3}(z, \tau)$, in the following form:

$$
\begin{gathered}
\left.\lambda_{1} \frac{\partial t_{1}(z, \tau)}{\partial z}\right|_{z=z_{1}}-\left.\lambda_{2} \frac{\partial t_{2}(z, \tau)}{\partial z}\right|_{z=z_{2}}=w \\
\left.\lambda_{1} \frac{\partial t_{1}(z, \tau)}{\partial z}\right|_{z=z_{1}}=\frac{1}{R}\left(t_{2}\left(z_{2}, \tau\right)-t_{1}\left(z_{1}, \tau\right)\right)+\frac{w}{2} .
\end{gathered}
$$

The obtained conditions allow one to close the problem of heat conduction for the layers (1), (2) and determine the functions of the temperature $t_{1}(z, \tau)$ and $t_{2}(z, \tau)$ from the equations (1) and conditions (3), (4), (12), (13) without determining the temperature in the thin inclusion.

In the process, the influence of the layer on the temperature field is taken into account by means of the coefficient of thermal contact resistance $R$ in the condition (13). According to the condition (13), we obtain that the temperature drop on the inner surfaces is proportional to the heat flux and to the coefficient of contact thermal resistance $R$. The heat flux under such formulation of the problem also features jump behavior and changes in the value of the power heat sources $w$ passing from the layer (1) to the layer (2).

If to neglect the thickness of the inclusion and direct its thickness to zero $h \rightarrow 0\left(z_{1} \rightarrow z_{*}\right.$, $\left.z_{2} \rightarrow z_{*} z_{*} \in\left(z_{1} ; z_{2}\right)\right)$, the effect of the inclusion on the heat transfer can be determined if we retain the coefficients $R$ and $w$ in the contact conditions, whose values are derived with account of the characteristics of the inclusion. Derived by passing through the limit, the conditions are called the nonideal heat transfer conditions.

\section{Heat transfer through thin inhomogeneous inclusion}

Consider the piecewise inhomogeneous conductive layer of the thickness $L$ composed of two layers contacting through a thin inclusion. To obtain different versions of nonideal contact conditions, the thin inclusion is considered to be heterogeneous, that is composed of two sublayers $\Omega_{3}=\left(z_{1} ; z_{*}\right)$ and $\Omega_{4}=\left(z_{*} ; z_{2}\right)$, in which there are heat sources of different capacities. With the help of the inclusion, thermophysical characteristics of which differ from the characteristics of the main layers, we simulate an effect of the interface presence on the peculiarities of heat transfer between the parts of the body.

Let us describe the process of heat exchange between the layers if in the inclusion in the domain $\Omega_{3}$, starting from a point of time heat sources of constant power $Q_{3}$ are acting, in the domain $\Omega_{4}$ - heat 
sources of constant power $Q_{4}$, and in the body's main parts of the domains $\Omega_{1}$ and $\Omega_{2}$ heat sources are absent, i.e. $Q_{1}=0, Q_{2}=0$. The coefficients of thermal conductivity and heat power for the main layers and thin inclusions we denote as $\lambda_{i}, c_{i}(i=\overline{1 ; 4})$.

On the inner surfaces $z=z_{1}, z=z_{*}$, and $z=z_{2}$, to establish the impact of the inclusion on heat transfer between the main layers, we impose the conditions of the ideal thermal contact, i.e. the equality of heat fluxes and of temperatures on both sides of the inclusion

$$
\begin{aligned}
& \left.\lambda_{1} \frac{\partial t_{1}(z, \tau)}{\partial z}\right|_{z=z_{1}}=\left.\lambda_{3} \frac{\partial t_{3}(z, \tau)}{\partial z}\right|_{z=z_{1}}, \quad t_{1}\left(z_{1}, \tau\right)=t_{3}\left(z_{1}, \tau\right) ; \\
& \left.\lambda_{3} \frac{\partial t_{3}(z, \tau)}{\partial z}\right|_{z=z_{*}}=\left.\lambda_{4} \frac{\partial t_{4}(z, \tau)}{\partial z}\right|_{z=z_{*}}, \quad t_{3}\left(z_{*}, \tau\right)=t_{4}\left(z_{*}, \tau\right) ; \\
& \left.\lambda_{4} \frac{\partial t_{4}(z, \tau)}{\partial z}\right|_{z=z_{2}}=\left.\lambda_{2} \frac{\partial t_{2}(z, \tau)}{\partial z}\right|_{z=z_{2}}, \quad t_{4}\left(z_{2}, \tau\right)=t_{2}\left(z_{2}, \tau\right) .
\end{aligned}
$$

Considering the small thickness of the inclusion, we use the linear approximate representations of its temperature functions $t_{3}(z, \tau), t_{4}(z, \tau)$ with respect to the thickness [8]. Considering also the influence of heat sources, in the domain $\Omega_{3}$ we can write down the temperature function in the form:

$$
t_{3}\left(z^{\prime}, \tau\right)=T_{1}^{\prime}(\tau)+\frac{z^{\prime}}{h} T_{2}^{\prime}(\tau)-\frac{Q_{3}}{\lambda_{3}} \frac{z^{\prime 2}}{2}
$$

where $T_{1}^{\prime}(\tau)$ and $T_{2}^{\prime}(\tau)$ are the average temperature with respect to the thickness $h_{3}=z_{*}-z_{1}$ and the temperature instant in $\Omega_{3}, z^{\prime}=z-\left(z_{1}+z_{*}\right) / 2$ is the changed coordinate $z$, that the origin $z^{\prime}$ to be in the middle of the interval $\left[z_{1}, z_{*}\right]$. In the domain $\Omega_{4}$, we use the same approximation of the temperature function

$$
t_{4}\left(z^{\prime \prime}, \tau\right)=T_{1}^{\prime \prime}(\tau)+\frac{z^{\prime \prime}}{h} T_{2}^{\prime \prime}(\tau)-\frac{Q_{4}}{\lambda_{4}} \frac{z^{\prime \prime 2}}{2},
$$

where $T_{1}^{\prime \prime}(\tau)$ and $T_{2}^{\prime \prime}(\tau)$ have the similar meaning for $h_{4}=z_{2}-z_{*}, z^{\prime \prime}=z-\left(z_{*}+z_{2}\right) / 2$.

Substituting the expressions (15), (16) into the conditions (14) of thermal contact on the inner surfaces. For $z=z_{1}$ we obtain

$$
t_{1}\left(z_{1}, \tau\right)=T_{1}^{\prime}(\tau)-\frac{1}{2} T_{2}^{\prime}(\tau)-\frac{Q_{3}}{\lambda_{3}} \frac{h_{3}^{2}}{8} ;\left.\quad \lambda_{1} \frac{\partial t_{1}(z, \tau)}{\partial z}\right|_{z=z_{1}}=\frac{\lambda_{3}}{h_{3}} T_{2}^{\prime}(\tau)+\frac{Q_{3} h_{3}}{2} ;
$$

for $z=z_{*}$ :

$$
T_{1}^{\prime}(\tau)+\frac{1}{2} T_{2}^{\prime}(\tau)-\frac{Q_{3}}{\lambda_{3}} \frac{h_{3}^{2}}{8}=T_{1}^{\prime \prime}(\tau)-\frac{1}{2} T_{2}^{\prime \prime}(\tau)-\frac{Q_{4}}{\lambda_{4}} \frac{h_{4}^{2}}{8} ; \quad \frac{\lambda_{3}}{h_{3}} T_{2}^{\prime}(\tau)-\frac{Q_{3} h_{3}}{2}=\frac{\lambda_{4}}{h_{4}} T_{2}^{\prime \prime}(\tau)+\frac{Q_{4} h_{4}}{2} ;
$$

for $z=z_{2}$ :

$$
t_{2}\left(z_{2}, \tau\right)=T_{1}^{\prime \prime}(\tau)+\frac{1}{2} T_{2}^{\prime \prime}(\tau)-\frac{Q_{4}}{\lambda_{4}} \frac{h_{4}^{2}}{8} ;\left.\quad \lambda_{2} \frac{\partial t_{2}(z, \tau)}{\partial z}\right|_{z=z_{2}}=\frac{\lambda_{3}}{h_{4}} T_{2}^{\prime}(\tau)-\frac{Q_{4} h_{4}}{2} .
$$

Form the conditions (17)-(19) we exclude $T_{1}^{\prime}(\tau), T_{2}^{\prime}(\tau), T_{1}^{\prime \prime}(\tau)$, and $T_{2}^{\prime \prime}(\tau)$. We obtain two following conditions

$$
\begin{gathered}
\left.\lambda_{1} \frac{\partial t_{1}(z, \tau)}{\partial z}\right|_{z=z_{1}}-Q_{3} h_{3}=\left.\lambda_{2} \frac{\partial t_{2}(z, \tau)}{\partial z}\right|_{z=z_{2}}+Q_{4} h_{4} \\
\left.\frac{\lambda_{1} h_{3}}{\lambda_{3}} \frac{\partial t_{1}(z, \tau)}{\partial z}\right|_{z=z_{1}}+\left.\frac{\lambda_{2} h_{4}}{\lambda_{4}} \frac{\partial t_{2}(z, \tau)}{\partial z}\right|_{z=z_{2}}=t_{2}\left(z_{2}, \tau\right)-t_{1}\left(z_{1}, \tau\right)+\frac{Q_{3} h_{3}^{2}}{2 \lambda_{3}}-\frac{Q_{4} h_{4}^{2}}{2 \lambda_{4}} .
\end{gathered}
$$


Note, from (17), (18), and (19) it also follows that the temperature differs on the boundaries of the inclusion $t_{2}\left(z_{2}, \tau\right)-t_{1}\left(z_{1}, \tau\right)=T_{2}^{\prime}(\tau)+T_{2}^{\prime \prime}(\tau)$. For the large $T_{2}^{\prime}(\tau)$ and $T_{2}^{\prime \prime}(\tau)$, the temperature drop can be quite noticeable even for a small value of the distance between $z=z_{1}$ and $z=z_{2}$.

Denote in the correlations (20), $(21)$ as $w_{i}=Q_{i} h_{i}(i=3 ; 4)$ the specific power of heat sources, $R_{i}=h_{i} / \lambda_{i}(i=3 ; 4)$ are contact thermal resistances. Let us write down the thermal contact conditions which take into account the influence of the inclusion on the temperature, but do not contain $t_{3}(z, \tau)$ and $t_{4}(z, \tau)$, in the following form:

$$
\begin{gathered}
\left.\lambda_{1} \frac{\partial t_{1}(z, \tau)}{\partial z}\right|_{z=z_{1}}-w_{3}=\left.\lambda_{2} \frac{\partial t_{2}(z, \tau)}{\partial z}\right|_{z=z_{2}}+w_{4} \\
\left.R_{3} \lambda_{1} \frac{\partial t_{1}(z, \tau)}{\partial z}\right|_{z=z_{1}}+\left.R_{4} \lambda_{2} \frac{\partial t_{2}(z, \tau)}{\partial z}\right|_{z=z_{2}}=t_{2}\left(z_{2}, \tau\right)-t_{1}\left(z_{1}, \tau\right)+\frac{w_{3} R_{3}}{2}-\frac{w_{4} R_{4}}{2} .
\end{gathered}
$$

They allow us to close the heat conduction problem for the main layers and to define the temperature functions $t_{1}(z, \tau)$ and $t_{2}(z, \tau)$ from the equation (1) and the conditions (3), (4), (22), and (23) without determining of the temperature function in the thin inclusion.

The influence of the inclusion on the temperature field is taken into account by means of the coefficients of thermal contact resistance $R_{3}, R_{4}$ in the condition (23), and heat sources - through the specific powers of heat sources $w_{3}, w_{4}$.

If we neglect the inclusion's thickness and direct the thickness $h_{3} \rightarrow 0, h_{4} \rightarrow 0\left(z_{1} \rightarrow z_{*}, z_{2} \rightarrow z_{*}\right)$, having remained the coefficients $R_{3}, R_{4}$ in the contact conditions, which values are obtained with taking into account the characteristics of the inclusion, and values of $w_{3}, w_{4}$, which are determined by the powers of internal heat sources, we obtain conditions called the conditions of nonideal heat transfer. A mathematical model using nonideal conditions of heat transfer does not involve a consideration of interlayers, and an interface is considered to be the surface $z=z_{*}$.

\section{Heat transfer problem with generalized nonideal heat transfer conditions}

In this Section we use conditions of nonideal heat transfer for the formulation of the problem of heat transfer between contacting two layers $\Omega_{1}=\left(0 ; z_{*}\right)$ and $\Omega_{2}=\left(z_{*} ; L\right)$. Denote $t(z, \tau)$ as the temperature function, $\lambda(z), c(z)$ are the coefficients of heat conductivity and of specific heat in a combined layer

$$
t(z, \tau)=\left\{\begin{array}{ll}
t_{1}(z, \tau), & z \in \Omega_{1} \\
t_{2}(z, \tau), & z \in \Omega_{2}
\end{array}, \quad \lambda(z)=\left\{\begin{array}{ll}
\lambda_{1}, & z \in \Omega_{1} \\
\lambda_{2}, & z \in \Omega_{2}
\end{array}, \quad c(z)=\left\{\begin{array}{ll}
c_{1}, & z \in \Omega_{1} \\
c_{2}, & z \in \Omega_{2}
\end{array} .\right.\right.\right.
$$

The heat equation in the domain $\Omega=\Omega_{1} \cup \Omega_{2}$ we write down as follows

$$
c(z) \frac{\partial t(z, \tau)}{\partial \tau}-\frac{\partial}{\partial z}\left(\lambda(z) \frac{\partial t(z, \tau)}{\partial z}\right)=0, \quad z \in \Omega, \quad \tau>0 .
$$

The initial condition has the form

$$
t(z, 0)=\varphi(z), \quad z \in \Omega .
$$

Let us impose the following conditions on the outer boundaries

$$
t(0, \tau)=f_{0}, \quad t(L, \tau)=f_{L} .
$$

The contact conditions (22), (23) for $z=z_{*}$ take the following form:

$$
\left.\lambda_{1} \frac{\partial t_{1}(z, \tau)}{\partial z}\right|_{z=z_{*}}-w_{3}=\left.\lambda_{2} \frac{\partial t_{2}(z, \tau)}{\partial z}\right|_{z=z_{*}}+w_{4}
$$

Mathematical Modeling and Computing, Vol. 2, No. 1, pp. 33-47 (2015) 


$$
\left.R_{3} \lambda_{1} \frac{\partial t_{1}(z, \tau)}{\partial z}\right|_{z=z_{*}}+\left.R_{4} \lambda_{2} \frac{\partial t_{2}(z, \tau)}{\partial z}\right|_{z=z_{*}}=t_{2}\left(z_{*}, \tau\right)-t_{1}\left(z_{*}, \tau\right)+\frac{w_{3} R_{3}}{2}-\frac{w_{4} R_{4}}{2} .
$$

According to the conditions (27), (28), the temperature function $t(z, \tau)$ and a heat flux for $z=z_{*}$ can have discontinuities of the first kind.

The solution of the contact-boundary problem (24), (25), (26), (27), and (28) - the function $t(z, \tau)$ and the heat flux for $z=z_{*}$ have abrupt change. Let us perform decomposition of the heat conduction linear problem. For this purpose, let us represent the unknown function $t(z, \tau)$ as the sum

$$
t(z, \tau)=t_{\varphi}(z, \tau)+t_{3}(z, \tau)+t_{w}(z, \tau)
$$

where $t_{\varphi}(z, \tau)$ is the solution of the heat equation for $w=0$ in the conditions (27), (28) which satisfies the initial condition (25) and has zero values on the boundaries $z=0$ and $z=L$ of the body, $t_{3}(z, \tau)$ is the solution of heat conduction problem with the boundary conditions (26), (27), and (28), a zero initial temperature and $w=0, t_{w}(z, \tau)$ is the temperature function of heat sources with zero boundary conditions and for $\tau=0$.

Each function included in the expression (29) is a solution of the heat equation. Let us obtain them using the method of separation of variables. Represent the function $t_{\varphi}(z, \tau)$ as a series

$$
t_{\varphi}(z, \tau)=\sum_{i=1}^{\infty} \sigma_{i} Z_{i}(z) \Theta_{i}(\tau)
$$

We substitute the term of the solution $Z_{i}(z) \Theta_{i}(\tau)$ - the product of functions of coordinates and time - into the heat equation (24). We divide both sides of the equation by $Z_{i}(z) \Theta_{i}(\tau)$ and we obtain the equality to find $Z_{i}(z)$ and $\Theta_{i}(\tau)(i=1,2, \ldots)$

$$
\frac{\dot{\Theta}_{i}(\tau)}{\Theta_{i}(\tau)}=\frac{1}{c(z) Z_{i}(z)} \frac{d}{d z}\left(\lambda(z) \frac{d Z_{i}(z)}{d z}\right) \equiv-\left(\frac{\pi}{L} \sqrt{\frac{\bar{\lambda}}{\bar{c}}} \eta_{i}\right)^{2} .
$$

The right side of the equality is written with a constant multiplier, which provides the desired scalability and dimension of the eigenvalues $\eta_{i}$. By means of $\bar{\lambda}$ and $\bar{c}$ we denote the average values of the coefficients of heat conductivity and heat capacity in the combined layer.

Let us find the solution of the equation

$$
\frac{1}{c(z) Z_{i}(z)} \frac{d}{d z}\left(\lambda(z) \frac{d Z_{i}(z)}{d z}\right)=-\left(\frac{\pi}{L} \sqrt{\frac{\bar{\lambda}}{\bar{c}}} \eta_{i}\right)^{2} .
$$

Eigenfunctions $Z_{i}(z)$, as well as the function $t_{\varphi}(z, \tau)$, satisfy zero conditions on the boundaries of the body

$$
Z_{i}(0)=0, \quad Z_{i}(L)=0
$$

and the conditions for $z=z_{*}$, which result from conditions (27), (28)

$$
\begin{gathered}
{\left[\lambda(z) \frac{d Z_{i}(z)}{d z}\right]_{z=z_{*}-0}=\frac{1}{R_{3}+R_{4}}\left(Z_{i}\left(z_{*}+0\right)-Z_{i}\left(z_{*}-0\right)\right) ;} \\
{\left[\lambda(z) \frac{d Z_{i}(z)}{d z}\right]_{z=z_{*}-0}=\left[\lambda(z) \frac{d Z_{i}(z, \tau)}{d z}\right]_{z=z_{*}+0} .}
\end{gathered}
$$


As a result, we obtain eigenfunctions of the corresponding Sturm-Liouville problem with a jump of the first kind for $z=z_{*}$

$$
Z_{i}(z)=\left\{\begin{array}{ll}
\sin \left(\frac{\pi \eta_{i}}{L} \gamma_{1} z\right), & z \in \Omega_{1} \\
-\frac{\lambda_{1} \gamma_{1} \cos \left(\frac{\pi \eta_{i}}{L} \gamma_{1} z_{*}\right)}{\lambda_{2} \gamma_{2} \cos \left(\frac{\pi \eta_{i}}{L} \gamma_{2}\left(L-z_{*}\right)\right)} \sin \left(\frac{\pi \eta_{i}}{L} \gamma_{2}(L-z)\right), & z \in \Omega_{2}
\end{array} .\right.
$$

Here we denote $\gamma_{j}=\sqrt{\frac{\lambda c_{j}}{\bar{c} \lambda_{j}}}(j=1 ; 2)$.

To find the eigenvalues $\eta_{i}$, we obtain the equation

$$
F\left(\eta_{i}\right)=0
$$

where

$$
\begin{aligned}
F(\eta)= & {\left[\frac{\pi \eta}{L} \lambda_{1} \gamma_{1} R \cos \left(\frac{\pi \eta}{L} \gamma_{1} z_{*}\right)+\sin \left(\frac{\pi \eta}{L} \gamma_{1} z_{*}\right)\right] \cos \left(\frac{\pi \eta}{L} \gamma_{2}\left(L-z_{*}\right)\right)+} \\
& +\frac{\lambda_{1} \gamma_{1}}{\lambda_{2} \gamma_{2}} \cos \left(\frac{\pi \eta}{L} \gamma_{1} z_{*}\right) \sin \left(\frac{\pi \eta}{L} \gamma_{2}\left(L-z_{*}\right)\right), \quad R=R_{3}+R_{4} .
\end{aligned}
$$

To estimate the values of the roots of the characteristic equation is possible due to the graph of the function $F(\eta)$ (Fig. 2). In the process of numerical solving the equation (37), it should be considered the accuracy of the calculations in order not to miss some roots, as it is seen in Fig. 2 they can have close values.

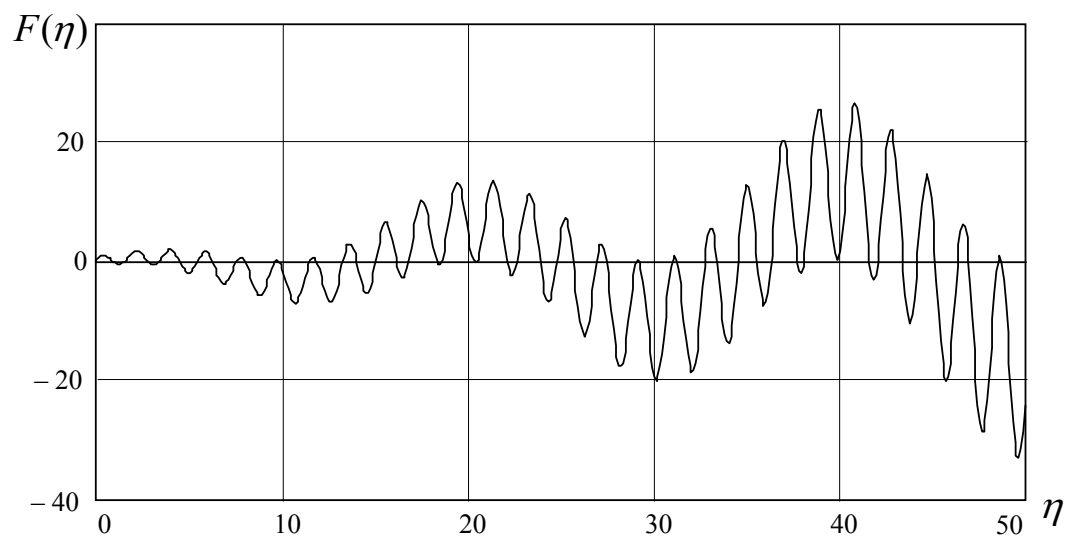

Fig. 2. Graphical representation of the roots of the characteristic equation (37).

Note that $\eta_{0}=0$ and $Z_{0}(z)=0$. The functions $Z_{i}(z)(i=1,2, \ldots)$ are orthogonal with the weight factor $c(z)$, i.e.

$$
\int_{0}^{L} c(z) Z_{k}(z) Z_{l}(z) d z\left\{\begin{array}{ll}
=0, & k \neq l \\
\neq 0, & k=l
\end{array} .\right.
$$

From the second equation of separation of variables

$$
\dot{\Theta}_{i}(\tau)+\left(\frac{\pi}{L} \sqrt{\frac{\bar{\lambda}}{\bar{c}}} \eta_{i}\right)^{2} \Theta_{i}(\tau)=0
$$


we obtain

$$
\Theta_{i}(\tau)=\exp \left[-\left(\frac{\pi}{L} \sqrt{\frac{\bar{\lambda}}{\bar{c}}} \eta_{i}\right)^{2} \tau\right] .
$$

Since $\Theta_{i}(0)=1$, then for $\tau=0$ the condition (25) can be reduced to the following one

$$
\varphi(z)=\sum_{i=1}^{\infty} \sigma_{i} Z_{i}(z)
$$

The coefficients $\sigma_{i}$ are obtained by expanding $\varphi(z)$ in terms of the system of orthonormal functions $Z_{i}(z) /\left\|Z_{i}(z)\right\|$. That is,

$$
\sigma_{i}=\frac{1}{\left\|Z_{i}(z)\right\|} \int_{0}^{L} \varphi(z) c(z) Z_{i}(z) d z
$$

where $\left\|Z_{i}(z)\right\|=\int_{0}^{L} c(z) Z_{i}^{2}(z) d z$. The resulting function ensures the $t(z, \tau)$ to hold the initial condition (25).

Other terms in the expression (29) we represent as the sum of the stationary and non-stationary solutions of the corresponding heat conduction problem, namely

$$
\begin{aligned}
& t_{3}(z, \tau)=t_{G}(z)+\sum_{i=1}^{\infty} \mu_{i} Z_{i}(z) \Theta_{i}(\tau), \\
& t_{w}(z, \tau)=t_{Q}(z)+\sum_{i=1}^{\infty} \nu_{i} Z_{i}(z) \Theta_{i}(\tau) .
\end{aligned}
$$

We determine coefficients $\mu_{i}, \nu_{i}$ as the coefficients of expansions for functions $t_{G}(z)$ and $t_{Q}(z)$ in terms of $Z_{i}(z)$ similarly as $\sigma_{i}$ by the formulae of the kind (41).

Substituting the expressions (30), (42), and (43) into the correlation (29), we obtain the formula for calculating the temperature $t(z, \tau)$

$$
t(z, \tau)=t_{G}(z)+t_{Q}(z)+\sum_{i=1}^{\infty}\left(\sigma_{i}+\mu_{i}+\nu_{i}\right) Z_{i}(z) \Theta_{i}(\tau) .
$$

This representation of the solution of the heat equation satisfies the initial (25) and boundary (26), (27), (28) conditions. For $\tau \rightarrow \infty$ we pass to the stationary state since $t(z, \tau) \rightarrow t_{G}(z)+t_{Q}(z)$.

\section{Individual cases of nonideal conditions of heat transfer (the study of solutions)}

Conditions of heat exchange (27), (28) can be used for obtaining the known and new conditions of nonideal heat transfer. Then the solution of the heat conduction problem (24)-(28) makes it possible to study the different ways of thermal contact of bodies - through thin layers with the heat dissipation and the heat absorption on the interface, and also with a combination of different factors.

\section{Heat insulating contact}

Most often, the nonideal conditions of heat exchange foresee the reflection of a significant change in temperature when passing the interface. To obtain such conditions from (27), (28), we assume that the surface heat sources are absent $\left(w_{3}=0, w_{4}=0\right)$, and the total thermal resistance of the inclusion 
$R=R_{3}+R_{4}$. Then contact conditions take the usual form:

$$
\left.\lambda_{1} \frac{\partial t_{1}(z, \tau)}{\partial z}\right|_{z=z_{*}}=\left.\lambda_{2} \frac{\partial t_{2}(z, \tau)}{\partial z}\right|_{z=z_{*}},\left.\quad \lambda_{1} \frac{\partial t_{1}(z, \tau)}{\partial z}\right|_{z=z_{*}}=\frac{1}{R}\left(t_{2}\left(z_{*}, \tau\right)-t_{1}\left(z_{*}, \tau\right)\right) .
$$

From these conditions it follows that the function of heat flux is continuous and the temperature function has a jump when passing the interface. The magnitude of the jump is proportional to the heat flux on the inner boundary and to the thermal resistance $R$.

To illustrate this, let us calculate the temperature function in a composite layer under nonideal conditions of heat transfer (45) for $z=z_{*}$. On the boundary $z=0$, the constant temperature is ensured $f_{0}=1$, on the other boundary $z=L$, the temperature is equal to zero $f_{L}=0$, and the initial distribution is as follows $\varphi(z)=0$. According to the formula (29), in this case $t(z, \tau)=t_{3}(z, \tau)$ since $t_{\varphi}(z, \tau)=0, t_{w}(z, \tau)=0$.

In Fig. 3 it is shown the graphs of the change in the temperature function $t_{3}(z, \tau)$ with respect to the layer thickness, which are obtained as a solution of the heat conduction problem (24)-(28) with taking into account the thermal resistance $R=0.01 \mathrm{~m}^{2} \cdot K / \mathrm{W}$ (solid curves) and without it; if $R=0$ (dashed curves); at some points of time $\tau=30 ; 900 ; 360 \mathrm{~s}$. With this, the layer parameters $L=0.025$; $z_{*}=0.011$, and the coefficients of thermal conductivity and heat capacity are the following: $\lambda_{1}=0.8$; $\lambda_{2}=0.5 \mathrm{~W} /(\mathrm{m} \cdot K) ; c_{1}=1.5 \cdot 10^{6} ; c_{2}=1 \cdot 10^{6} \mathrm{~J} /\left(\mathrm{m}^{3} \cdot K\right)$.

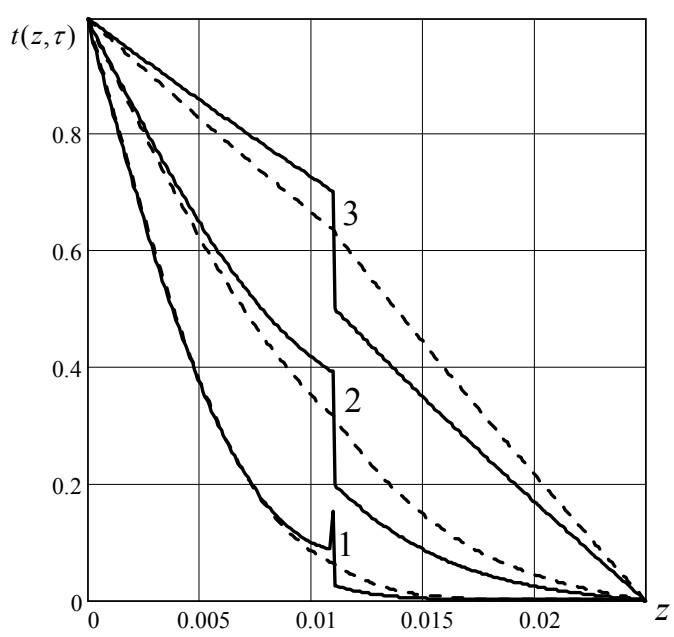

Fig. 3. Changes in temperature with respect to the thickness under nonideal conditions of heat transfer (45) between the layers. Curves $1-3$ correspond to the points of time $\tau=30 ; 90 ; 360 \mathrm{~s}$.

As we would expect, in the case of replacement of the ideal contact conditions with the nonideal ones under the exclusion of the sublayer of the contact from consideration, the behavior of the temperature function becomes to be discontinuous. In the real world conditions, the value of the jump increases with the increase in temperature and it must take the maximum value in the stationary case, the use of the nonideal contact conditions (45) causes a jump proportional to the heat flux at a constant thermal resistance. Therefore, at low levels of temperature, the solution of the problem has unnatural character. Passing through the interface, the peak is formed (curve 1). This is due to the replacement of the ideal conditions of heat transfer in a layer with the inclusion with the nonideal conditions of heat transfer in a layer without the inclusion, as well as due to the summation of series expansion (42) of the temperature function with respect to its eigenfunctions $Z_{i}(z)$.

\section{Nonideal thermal contact under heating}

The second frequently used form of nonideal thermal contact conditions requires a presence of surface heat sources. Let us obtain such conditions assuming that in the formulae (27), (28) the surface heat sources $w_{3}=w_{4}\left(w=w_{3}+w_{4}\right)$, and the total thermal resistance of the inclusion $R_{3}=R_{4}$ $\left(R=R_{3}+R_{4}\right)$. Then contact conditions have their usual form:

$$
\left.\lambda_{1} \frac{\partial t_{1}(z, \tau)}{\partial z}\right|_{z=z_{*}}=\left.\lambda_{2} \frac{\partial t_{2}(z, \tau)}{\partial z}\right|_{z=z_{*}}+w,\left.\quad \lambda_{1} \frac{\partial t_{1}(z, \tau)}{\partial z}\right|_{z=z_{*}}=\frac{1}{R}\left(t_{2}\left(z_{*}, \tau\right)-t_{1}\left(z_{*}, \tau\right)\right)+\frac{w}{2} .
$$


The heat flux and the temperature function passing the interface are changed abruptly. Here the magnitude of the flux jump is constant and equals $w$. The temperature jump is proportional to the total heat flux, which includes the term of the surface sources.

Calculate the function of the temperature field in the composite layer under the conditions of inner heat transfer (46), on the boundaries $z=0, z=L$ of which the temperature is zero. The initial distribution $\varphi(z)=0$. According to the formula (29), in this case $t(z, \tau)=t_{w}(z, \tau)$ since $t_{\varphi}(z, \tau)=$ $=0, t_{3}(z, \tau)=0$. We assume that the total surface specific power of heat sources $w=100 \mathrm{~W} / \mathrm{m}^{2}$. The interface $z_{*}=0.011 \mathrm{~m}$ causes the thermal resistance $R=0.01 \mathrm{~m}^{2} \mathrm{~K} / \mathrm{W}$. Other parameters and characteristics of the layer are the same as in the previous case.

Fig. 4 shows graphs of the function $t_{w}(z, \tau)$. The temperature jump of an increase or a decrease depends on thermal conductivity of the contacting layers. If $\lambda_{1}>\lambda_{2}$, then passing from the layer 1 to the layer 2, the temperature will increase (Fig. 4). Otherwise $\lambda_{1}<\lambda_{2}$, the temperature will decrease abruptly. Obtaining the temperature function by means of summation of the series (43) leads to the characteristic abnormalities of the jump.

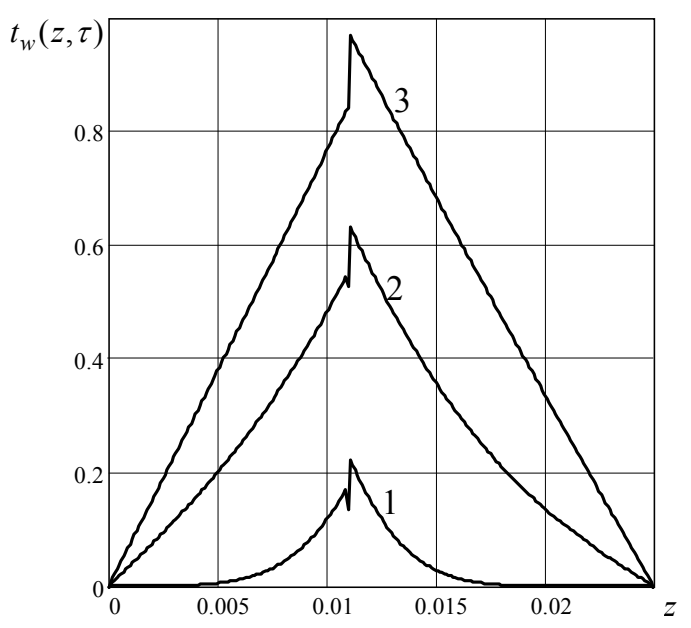

Fig. 4. Temperature distributions along the thickness of the composite layer under heating by inner heat sources. Curves $1-3$ correspond to the points of time $\tau=30 ; 60 ; 360 \mathrm{~s}$.

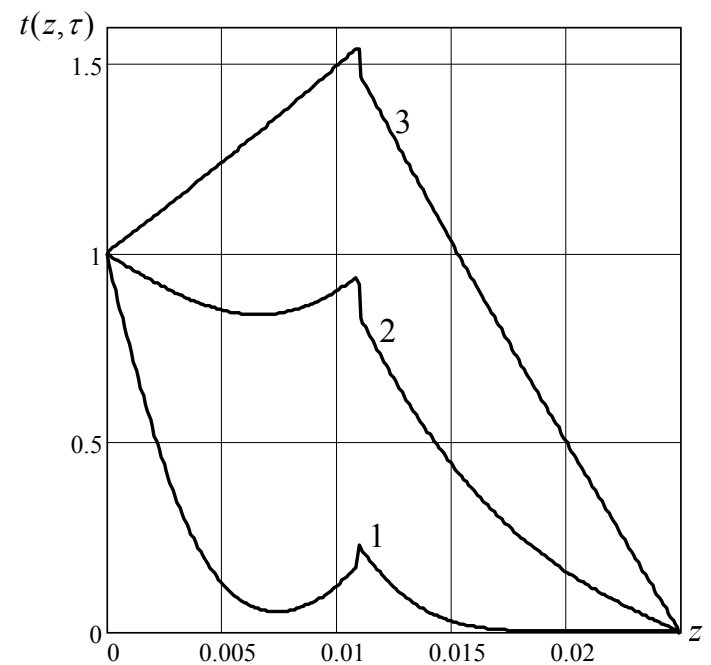

Fig. 5. Change of the temperature along the thickness of the composite layer for $\lambda_{1}=0.8, \lambda_{2}=0.5$, $w=100 \mathrm{~W} / \mathrm{m}^{2}$. Curves $1-3$ correspond to the points of time $\tau=10 ; 90 ; 360 \mathrm{~s}$.

Fig. 5 demonstrates graphs of the function $t(z, \tau)$, which is a sum of functions $t_{3}(z, \tau)$ (heating from the surfaces $f_{0}=1, f_{L}=0$ ) and $t_{w}(z, \tau)$ (heating by inner heat sources $w=100 \mathrm{~W} / \mathrm{m}^{2}$ ). For the parameters and layer characteristics taken for this calculations, the jump of a decrease of a function is completely offset by a jump of an increase of another function. In this case, the abrupt change in temperature cannot be observed experimentally. However, the behavior of the temperature function (especially for small values of time) indicates the interface influence on it.

If we change the characteristics of the contacting layers or place on the surface heat sinks instead of the heat sources, the temperature function jump will increase with the superposition of two drop-down functions $t_{3}(z, \tau)$ and $t_{w}(z, \tau)$. Fig. 6 illustrates graphs of the function $t(z, \tau)$ in a layer with another coefficients of thermal conductivity of the contacting layers, and in Fig. 7 we have negative heat sources on the interface.

It should be also noted that the function of the heat flux has a jump of the magnitude $w$ and transiting to the stationary state it takes a constant value in the domain $\Omega_{1}$ and a constant value in the domain $\Omega_{2}$. 


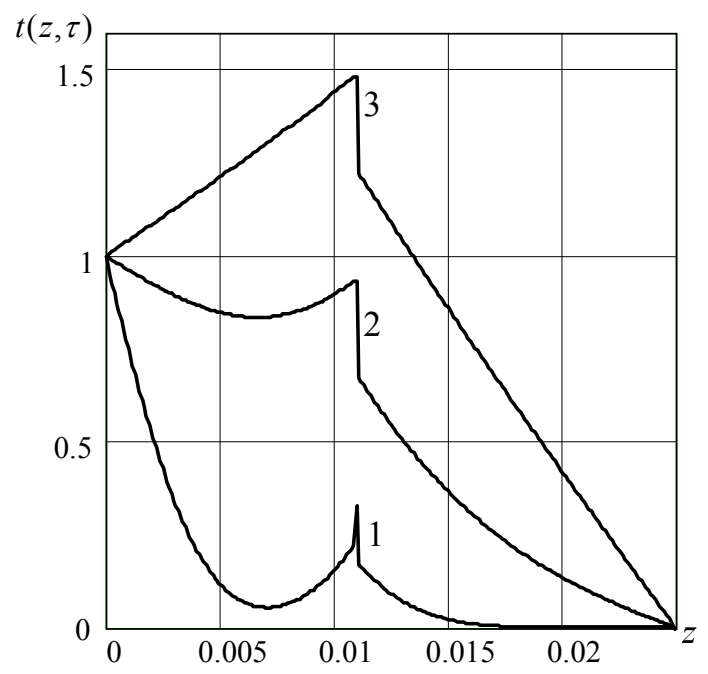

Fig. 6. Temperature distributions along the thickness of the composite layer for $\lambda_{1}=0.5, \lambda_{2}=0.8$, $w=100 \mathrm{~W} / \mathrm{m}^{2}$. Curves $1-3$ correspond to the points of time $\tau=10 ; 90 ; 360 \mathrm{~s}$.

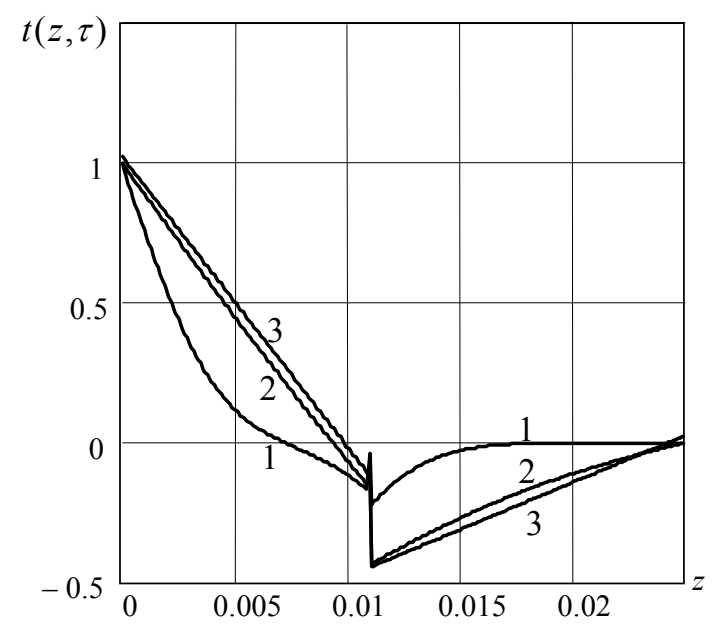

Fig. 7. Temperature distributions along the thickness of the composite layer for $\lambda_{1}=0.5, \lambda_{2}=0.8$, $w=-100 \mathrm{~W} / \mathrm{m}^{2}$. Curves $1-3$ correspond to the points of time $\tau=10 ; 90 ; 360 \mathrm{~s}$.

\section{Contact with heating one sublayer and cooling the other one}

Let in one part of the inclusion the heat sink (sinks) occurs, and in the other part - heat sources of the same intensity are acting. To obtain the conditions of thermal contact, we assume that $w_{3}=-w_{*}$, $w_{4}=w_{*}$. Total power of surface sources $w_{3}+w_{4}=0$ since $w_{3}=-w_{4}$. The contact conditions (27), (28) with denoting $R=R_{3}+R_{4}$, in this case we write down in the form:

$$
\left.\lambda_{1} \frac{\partial t_{1}(z, \tau)}{\partial z}\right|_{z=z_{*}}=\left.\lambda_{2} \frac{\partial t_{2}(z, \tau)}{\partial z}\right|_{z=z_{*}},\left.\quad \lambda_{1} \frac{\partial t_{1}(z, \tau)}{\partial z}\right|_{z=z_{*}}=\frac{1}{R}\left(t_{2}\left(z_{*}, \tau\right)-t_{1}\left(z_{*}, \tau\right)\right)-w_{*} .
$$

Since the heat sources and heat sinks completely offset each others, the heat fluxes are the same on both sides of the inner surface and the temperature abruptly increases passing the interface. The solution of the heat conduction problem (24), (25), (26), (47) we present in the form (29).

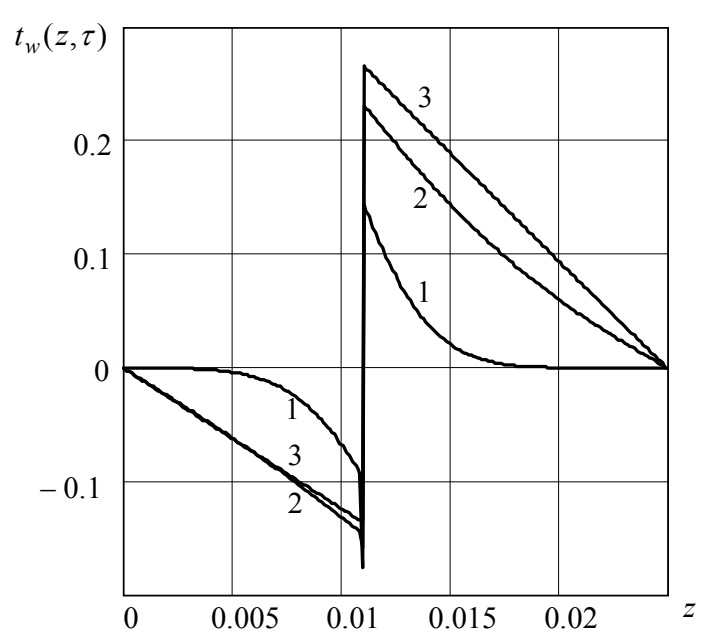

Fig. 8. Temperature changes along the thickness of the composite layer. Heat sinks and heat sources are in the vicinity of the interface. Curves 1-3 correspond to the time $\tau=10 ; 90 ; 360 \mathrm{~s}$.
If on the contrary, first the heat sources act, and then there is heat dissipation, the last term in the condition (47) is positive, and temperature has an abrupt decrease on the interface along the axis $z$.

A calculation of the function of the temperature field in the composite layer under nonideal conditions (47) is carried out. The initial distribution $\varphi(z)=0$, that causes $t_{\varphi}(z, \tau)=0$.

The interface $z_{*}=0.011 \mathrm{~m}$ causes the thermal resistance $R=0.01 \mathrm{~m}^{2} \cdot \mathrm{K} / \mathrm{W}$ and here heat sinks and heat sources are acting so that $w_{3}=-100 \mathrm{~W} / \mathrm{m}^{2}, w_{4}=100 \mathrm{~W} / \mathrm{m}^{2}$.

Fig. 8 shows graphs of changes of the temperature function $t_{w}(z, \tau)$ with respect to the thickness of the layer, which are obtained at the instances of time $\tau=10 ; 90 ; 360$ s for zero temperatures on the external boundaries $f_{0}=0$, 
$f_{L}=0$. Abrupt increase in temperature at its fall-off in the direction away of the boundary $z=0$ to the surface $z=L$ and with continuous flow of heat caused by the nature of heat sinks and heat sources.

In Fig. 9, the graphs of the function $t(z, \tau)$ are shown respectively for the case of the presence of heating from the boundary $z=0 f_{0}=1\left(f_{L}=0\right)$.

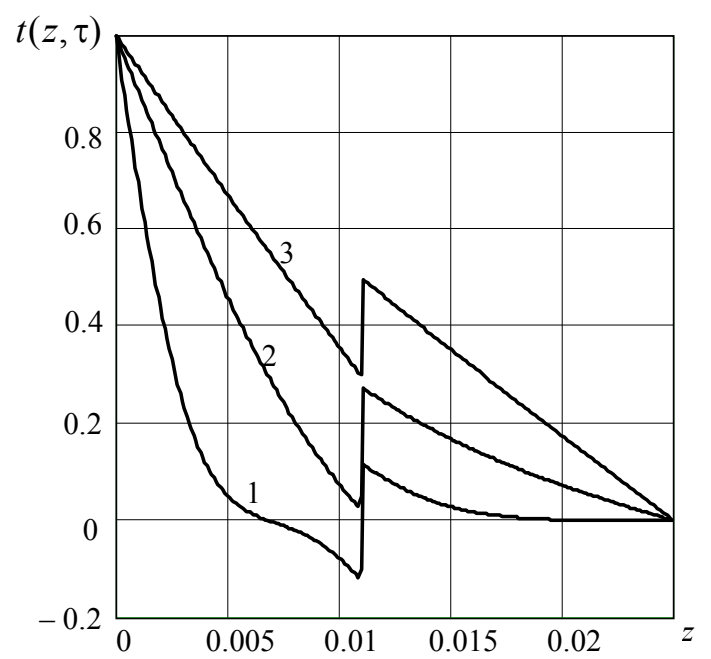

Fig. 9. Temperature changes along the thickness of the composite layer. Heat sinks and heat sources are in the vicinity of the interface. Curves 1-3 correspond to the time $\tau=10 ; 90 ; 360 \mathrm{~s}$.

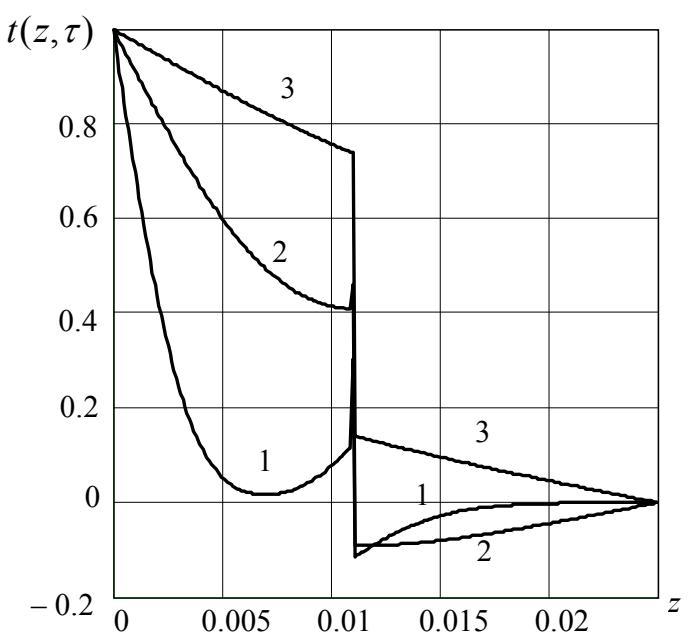

Fig. 10. Temperature changes along the thickness of the composite layer. Heat sinks and heat sources are in the vicinity of the interface. Curves 1-3 correspond to the time $\tau=10 ; 90 ; 360 \mathrm{~s}$.

\section{Conclusions}

Connecting flat heat conducting layers so that in the vicinity of the contact a "perturbation of the material" with the insulating effect occurs and heat sources (sinks) also are raised, the conditions of various kinds of contact heat transfer between the layers are obtained by modeling of heat transfer through a thin sublayer. Assuming that its thermal characteristics can differ significantly from the characteristics of the main layers, we observe considerable temperature drops, which are modeled by an abrupt function. The presence of heat sources in the contact zone leads to a jump of the temperature function and the heat flux. Depending on the sequence of distribution of heat sources and heat sinks, the jump can be toward the temperature increase as well as towards the temperature decrease. These effects are fully taken into account and represented by nonideal conditions of heat exchange between contacting layers. The value of thermal resistance (which is included into these conditions) is suggested to be determined by means of values more usual to measurement, e.g. thermal conductivity and the thickness of inclusion. Surface specific power of heat sources can be set if the magnitude of heat dissipation in the vicinity of the contact interface is known.

[1] Belyaev V., Berlov O., Klim V. Determination of heat transfer parameters of multilayer elements of constructions. 8 154-163 (2012). (In Ukrainian)

[2] Veselovsky V., Berlov A. Temperature fields of multilayer structural elements under the influence of fields of different physical nature. Metallurgical Heat Engineering. 1, n.16, 21-33 (2009). (In Russian)

[3] Mesnyankin S., Vikulov A., Vikulov D. Modern view on the problems of solids thermal contact. Uspekhi Fizicheskikh Nauk. 179, n.9, 495-970 (2009). (In Ukrainian) 
[4] Aleksandrov V., GubarevE. The problem of the interaction of coated bodies under deterioration, heat dissipation, and with taking into account the dependence of the coefficient of friction on temperature. Ecological Bulletin of the BSEC research centers. 25, 10-15 (2006). (In Russian)

[5] Veselovsky V. Contact thermal resistance in elements of constructions. Hydrodynamics and Heat and Mass Transfer Processes. Kyiv: Naukova Dumka. 120-125 (1986). (In Russian)

[6] Veselovsky V., Datsenko I., Nikulyikova V., Lyashenko V. Determination of unsteady boundary conditions on the fire walls of the houses on the basis of experimental measurements of temperatures. Visnyk of Dnipropetrovsk University. Ser. Mechanics. 6, n.1, 102-110 (2002). (In Russian)

[7] Veselovsky V., Ostrovskaya A., Lyashenko V., Belyi N., Stolbchenko V. Contact thermal resistance in structural elements. Journal of Engineering Physics. 3, 91-98 (2001). (In Russian)

[8] Pidstryhach Y. Selected Works. Kyiv: Naukova Dumka (1995). (In Ukrainian)

[9] Gera B. Modelling of nonideal heat transfer of contacting heat-conducting layers. Physical Modeling and Information Technology. 16, 52-60 (2012). (In Ukrainian)

[10] Gera B. Mathematical modeling of the conditions of nonideal thermal contactof layers through thin inclusion with heat sources. Physical Modeling and Information Technology. 18, 61-72 (2013). (In Ukrainian)

[11] Belyakov N., Noscko A. Nonideal thermal contact of bodies under friction (2010).

[12] Belyakov N., Noscko A. Mathematical modeling of thermal processes of friction under nonideal contact. Thermophysics of High Temperature. 47, n.1, 129-136 (2009). (In Russian)

[13] Belyakov N. S., NoskovA.P. Mathematical Simulation of Thermal Friction Processes under Conditions of Nonideal Contact. Heat and Mass Trasfer and Physical Gasdynamics. 46, n.1, 129-136 (2009).

[14] Aleksandrov V, GubarevE. Accounting for non-linear friction, deterioration, and heat dissipation from friction under nonideal thermal contact in the problem of the interaction of bodies with coating. Friction and deterioration. 25, n.5, 455-460 (2004). (In Russian)

[15] Aleksandrov V., GubarevE. Solving thermoelastic contact problems for cylindrical and spherical plain bearings. Friction and deterioration. 26, n.4, 347-357 (2005). (In Russian)

[16] Berlov O. Temperature fields of composite structures under the influence of sources of electromagnetic origin. Mashynoznavstvo. 4, n.142, 10-17 (2009). (In Ukrainian)

[17] Veselovsky V., Klim V., Belyi N. Modeling the influence of fields of different physical nature on the thermal regimes of structural elements of constructions. Visnyk of Dnipropetrovsk University. Ser. Mechanics. 6, n.1, 111-119 (2002). (In Russian) 


\title{
Отримання і дослідження умов теплового обміну через неоднорідне включення 3 джерелами тепла
}

\author{
Гера Б. В. ${ }^{1}$, Дмитрук В. А. ${ }^{2,3}$ \\ ${ }^{1}$ Дніпропетровсъкий націоналъний університет залізничного транспорту ім. академіка В. Лазаряна \\ вул. Іванни Блажкевич, 12а, 79052, Лъвів, Україна \\ ${ }^{2}$ Центр математичного моделювання IППММ ім. Я. С. Підстригача НАН Украӥни \\ вул. Дж. Дудаєва, 15, 79005, Львів, Україна \\ ${ }^{3}$ Національний університет «Львівсъка політехніка» \\ вул. С. Бандери, 12, 79013, Лъвів, Украӥна
}

Отримано умови неідеального теплообміну для задачі теплопровідності контактуючих через неоднорідне тонке включення між шарами, у якому діють джерела (стоки) тепла. У ці умови входять як термічні опори складових включення, так і питомі потужності джерел тепла у них. На цій основі записано задачу теплопровідності 3 умовами неідеального теплообміну без розгляду проміжного включення. Їх використання забезпечує отримання спостережуваних особливостей температури і теплового потоку, а саме функції температури і теплового потоку зазнають стрибкоподібної зміни при переході через поверхню контакту. Значення термічного опору пропонується визначати через більш звичні для вимірювання - коефіцієнт теплопровідності та товщину включення. Поверхневі питомі джерела тепла можна задати, якщо відомі величини тепловиділення на границі контакту. 3 отриманих узагальнених умов теплообміну можна отримати відомі умови неідеального теплового контакту як окремі випадки.

Ключові слова: задача теплопровідності, складений шар, тонке включення, теплоперенесення, умови неідеального контакту

2000 MSC: 80A20; 93A30; 35K05

УДК: $536.2: 539.3$ 